\title{
NEW SINGLE-RESISTOR CONTROLLED SINUSOIDAL OSCILLATOR CIRCUIT USING UNITY-GAIN CURRENT FOLLOWERS
}

\author{
MUHAMMAD TAHER ABUELMA'ATTI* and \\ HASSAN AL-DAGHREER
}

King Fahd University of Petroleum and Minerals, Box 203 Dhahran 31261 Saudi Arabia

(Received 25 May 1996; In final form 30 August 1996)

\begin{abstract}
A new configuration for realizing current-follower-based sinusoidal oscillator is presented. The circuit uses two unity-gain current followers, three capacitors, and three resistors, and enjoys independent control of its frequency and condition of oscillation. Experimental results are included.
\end{abstract}

\section{INTRODUCTION}

At present there is a growing interest in designing filters and oscillators using unity-gain current and voltage followers (Zele, Allstot and Fiez, 1993, Ramirez Angulo and Sanchez-Sinencio, 1994, Tsividis and Papananos, 1994, Celma, Sabadell and Martinez, 1995, Abuelma'atti, 1995a,b). This is attributed to the fact that unity-gain current and voltage follower circuits are simple compared to the conventional operational amplifier and other complicated active elements, which require large areas on the chip and consume relatively large powers. Of particular interest here is the design of RC-sinusoidal oscillators using unity-gain current followers. While a number of such oscillator circuits have been reported (Abuelma'atti, 1995a,b), unfortunately none of them enjoy the attractive

\footnotetext{
${ }^{*}$ Corresponding author.
} 
feature of independent control of the frequency and the condition of oscillation. The realization of sinusoidal oscillators using unity-gain currentfollowers and enjoying independent control of the frequency and the condition of oscillation would be attractive as they can provide variablefrequency oscillations using a small area on the chip and consuming relatively low power.

\section{PROPOSED CIRCUIT}

Consider the general circuit shown in Fig. 1. Assuming ideal currentfollowers with $i_{x}=i_{y}$ and $v_{x}=0$, routine analysis yields the characteristic equation given by

$$
Y_{3}\left(Y_{4}\left(Y_{1}+Y_{2}+Y_{5}\right)+Y_{2} Y_{5}\right)+Y_{2} Y_{5} Y_{6}=0
$$

If we choose

$$
\begin{gathered}
Y_{1}=G_{1}=1 / R_{1}, Y_{2}=s C_{2}, Y_{3}=G_{3}=1 / R_{3}, Y_{4}=G_{4}=1 / R_{4}, Y_{5}=s C_{5}, Y_{6} \\
=s C_{6}
\end{gathered}
$$

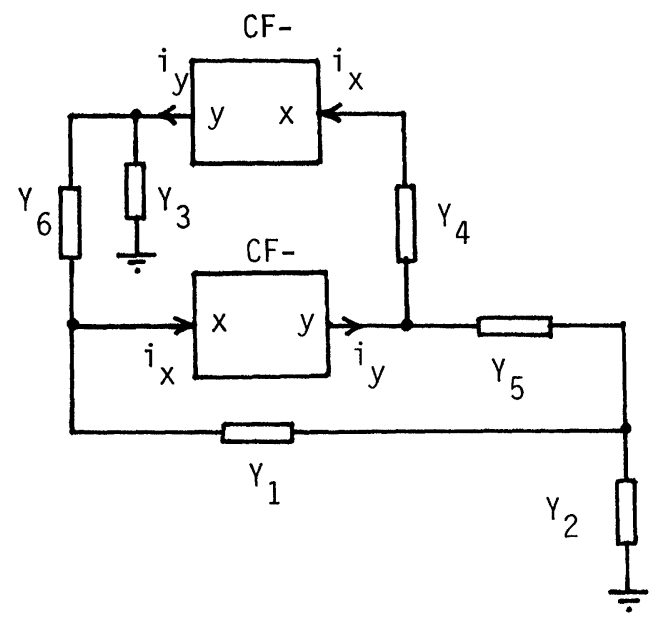

FIGURE 1 Proposed oscillator configuration 
(1) reduces to

$$
G_{3}\left(G_{4}\left(G_{1}+s\left(C_{2}+C_{5}\right)\right)+s^{2} C_{2} C_{5}\right)+s^{3} C_{2} C_{5} C_{6}=0
$$

Therefore, the frequency and the condition of oscillation can be expressed by

$$
\omega_{o}^{2}=\frac{\mathrm{G}_{1} \mathrm{G}_{4}}{\mathrm{C}_{2} \mathrm{C}_{5}}
$$

and

$$
G_{1} C_{6}=G_{3}\left(C_{2}+C_{5}\right)
$$

From (3) and (4), one can easily see that the frequency of oscillation can be adjusted by changing the conductance $G_{4}=1 / R_{4}$ without disturbing the condition of oscillation and the condition of oscillation can be adjusted by changing the condutance $G_{3}=1 / R_{3}$ without disturbing the frequency of oscillation. Thus, the circuit enjoys independent control of the frequency and the condition of oscillation.

\section{EXPERIMENTAL RESULTS}

Although there are several ways to implement the current-followers required for testing the proposed oscillator circuit, the test was performed using the AD844 positive-type second-generation currentconveyor(CCII + ) configured as as unity-gain current-follower with its high impedance input terminal grounded. Two CCII + can be configured to provide a minus-type current-follower. Obviously, this is not the simplest unity-gain current-follower realization. However, the kernel of the work presented in this paper is independent of the current-follower realization selected. Figure 2 shows the results obtained with $R_{1}=11 \mathrm{k} \Omega, R_{3}=20 \mathrm{k} \Omega$, $C_{2}=C_{5}=1.2 n F$, and $C_{6}=2.2 n F$. From Fig. 2, one can see that the experimental results are in excellent agreement with the theory presented. 


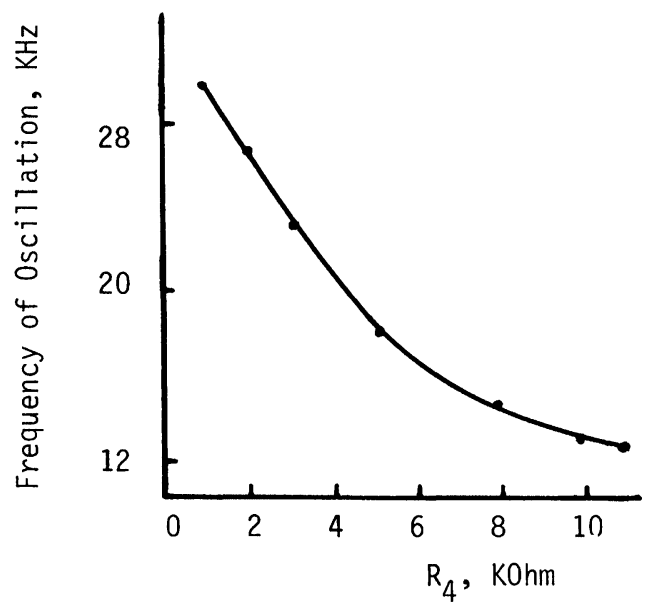

FIGURE 2 Measured (.) and calculated (-) frequency of oscillation of the circuit obtained from Fig. 1 with: $C_{2}=C_{5}=1.2 n F, C_{6}=2.2 n F R_{1}=11 \mathrm{k} \Omega, R_{3}=20 \mathrm{k} \Omega$.

However, as the frequency increases the discrepancies between measured and calculated frequencies of oscillation increase. This is attributed to the current-follower parasitics.

\section{CONCLUSION}

In this paper, a new current-follower-based sinusoidal oscillator circuit has been presented. The circuit uses two unity-gain current followers, three capacitors, and three resistors. In contrast with available current-follwerbased sinusoidal oscillators, the proposed ciruit enjoys the attractive feature of independent control of the frequency and the condition of oscillation. It is worth mentioning that another oscillator circuit, enjoying the same atrractive features, can be obtained by interchanging the capacitors and resistors.

\section{References}

[1] Abuelma'atti, M. T. (1995a). New current-mode oscillators using unity-gain current followers, Active and Passive Electronic Components, Vol. 18, pp. 159-164.

[2] Abuelma'atti, M. T. (1995b). New current-mode oscillators using a single unity-gain current follower, Active and Passive Electronic Components, Vol. 18, pp. 151-157. 
[3] Celma, C., Sabadell, J. and Martinez, P. (1995). Universal filter using unity-gain cells, Electronics Letters, Vol. 31, pp. 1817-1818.

[4] Ramirez Angulo, J. and Sanchez-Sinencio, E. (1994). Two approaches for currentmode filters using voltage follower and transconductance multipliers building blocks, IEE International Symposium on Circuits and Systems, Vol. 5, pp. 669-672.

[5] Tsividis, Y. and Papananos, Y. (1994). Continuous-time filters using buffers with gain lower than unity, Electronics Letters, Vol. 30, pp. 629-630.

[6] Zele, R. H., Allstot, D. J. and Fiez, T. S. (1993). Fully balanced CMOS current-mode circuits, IEEE Journal of Solid-State Circuits, Vol. 28, pp. 569-575. 

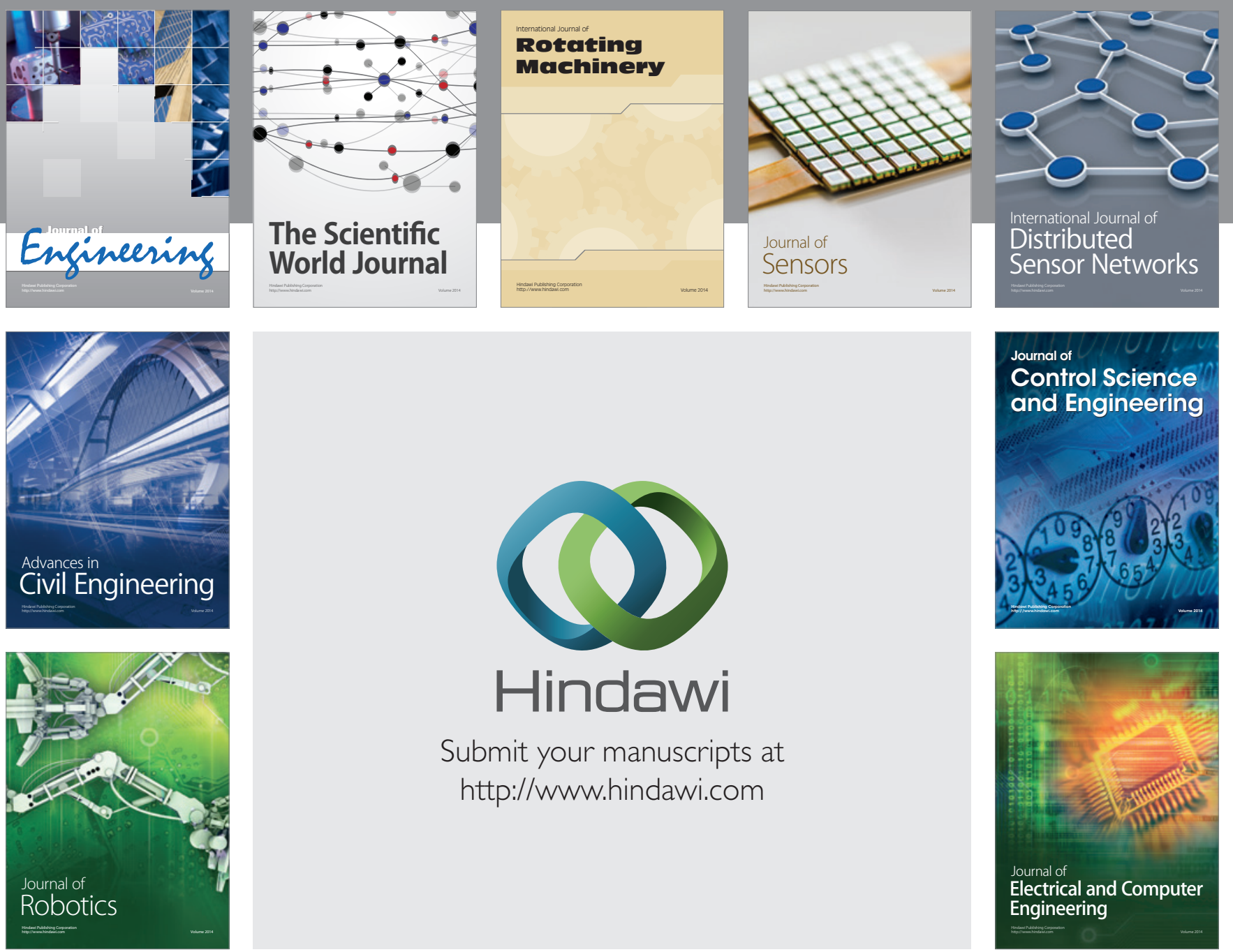

Submit your manuscripts at

http://www.hindawi.com
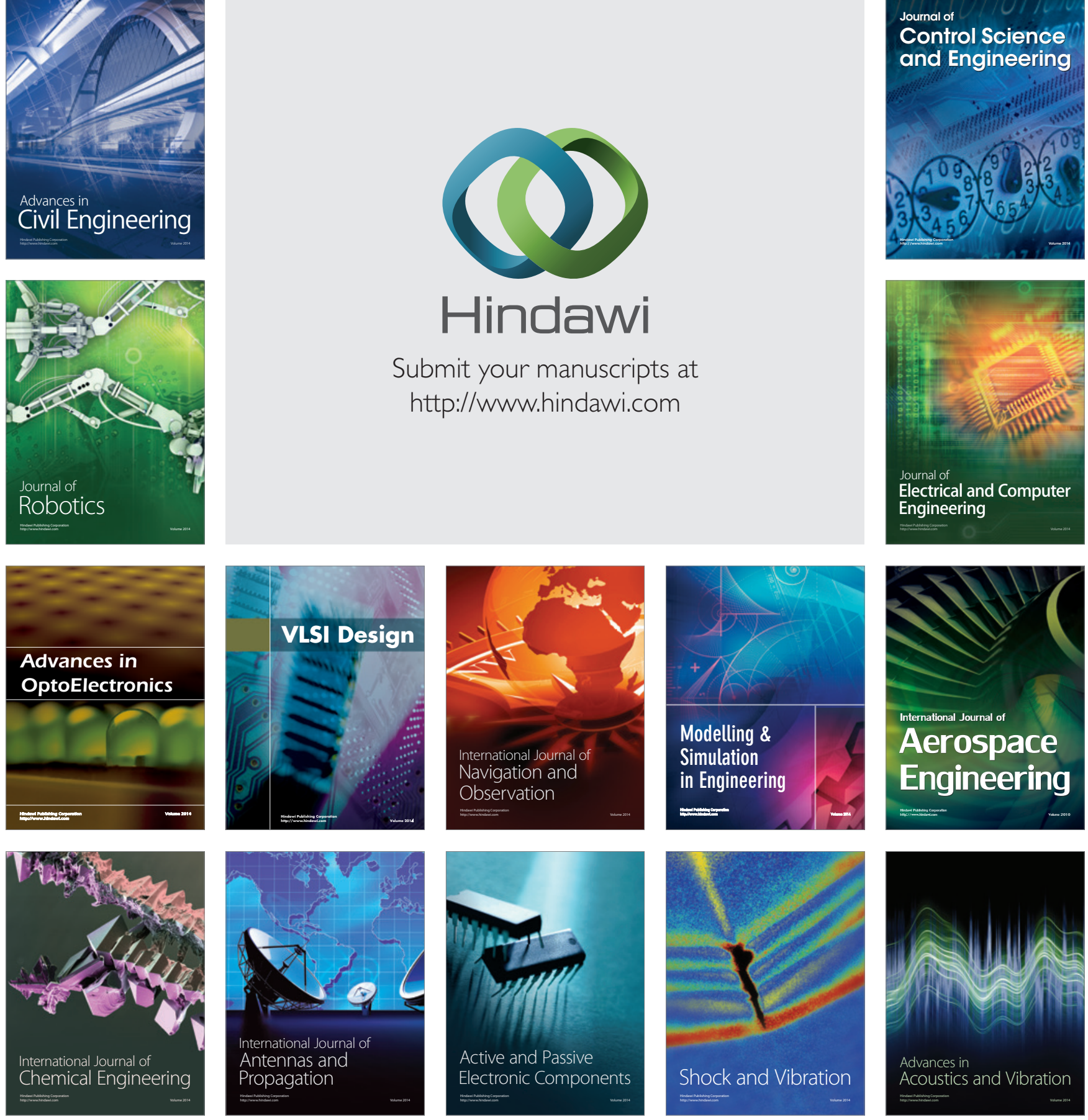